\title{
Cólon Biliar: Um Caso Raro de Complicação da Colelitíase
}

\section{Biliary Colon: A Rare Cholelithiasis complication}

Paulo Costa Correia ${ }^{1 *}$, Liliana Coutinho Cabral², Pedro Caldes², Maria Luís Santos ${ }^{3}$, Francisco O. Vieira ${ }^{3}$

*Autor Correspondente/Corresponding Author: Paulo Costa Correia [paulo.correia@ulsguarda.min-saude.pt]

\section{RESUMO}

A fístula colecistocólica é uma complicação rara da litíase biliar. A apresentação clínica é variável e inespecífica.

Os autores descrevem um caso clínico de uma paciente com obstrução mecânica baixa provocada pela impactação no cólon sigmoide de um cálculo biliar procedente de uma fístula colecistocólica.

Perante a tentativa infrutífera de remoção do cálculo através da colonoscopia, procedeu-se a laparotomia, com enterotomia após mobilização do cálculo e remoção do mesmo e colostomia lateral, sem intercorrências intraoperatórias. A eleição da técnica cirúrgica e o prognóstico são inevitavelmente condicionados pelo estado geral e comorbilidades associadas.

PALAVRAS-CHAVE: Doenças da Vesícula Biliar; Doenças do Cólon; Fístula Biliar; Fístula Intestinal

1. Assistente Graduado Sénior de Cirurgia Geral da ULS - Guarda / Prof. Auxiliar Convidado de Cirurgia da Faculdade de Ciências Médicas da Universidade da Beira Interior. 2. Assistente Hospitalar de Cirurgia Geral da ULS - Guarda, Portugal. 3. Interno do Ano Comum da ULS - Guarda, Portugal.

(Serviço de Cirurgia Geral do Hospital Sousa Martins - ULS Guarda, Portugal.)

• Autor (es) (ou seu (s) empregador (es)) e Gazeta Médica 2021. Reutilização permitida de acordo com CC BY-NC. Nenhuma reutilização comercial. ${ }^{\circledR}$ Author(s) (or their employer(s)) and Gazeta Médica 2021. Re-use permitted under CC BY-NC. No commercial re-use. 


\section{ABSTRACT}

Cholecystocolic fistula is a rare complication of biliary lithiasis. Clinical presentation is variable and nonspecific.

The authors describe a clinical case of a patient with low mechanical obstruction caused by impaction in the sigmoid colon of a gallstone from a cholecystolic fistula.

In view of the unsuccessful attempt to remove the calculus through colonoscopy, a laparotomy, with enterotomy was performed after mobilization of the calculus and removal of the calculation and lateral colostomy, without intraoperative complications.

The choice of the surgical technique and the prognosis are inevitably conditioned by the general state and associated co-morbidities.

KEYWORDS: Biliary Fistula; Colonic Diseases; Gallbladder Diseases; Intestinal Fistula

\section{INTRODUÇÃO}

As fístulas bilioentéricas foram descritas em 1890 por Courvoisier, sendo identificadas em $0,5 \%$ a $4,8 \%$ das cirurgias da vesícula/vias biliares.

São colecisto-cólicas em 8\% - 26,5\% dos casos (mais frequentemente a nível do ângulo hepático). Geralmente são diagnosticadas incidentalmente durante a colecistectomia. ${ }^{1-3}$ Os mecanismos fisiopatológicos implicados na formação deste tipo de fístulas baseiam-se em: alterações da perfusão/fenómenos de inflamação, pressão exercida pelos cálculos na parede da vesícula biliar. ${ }^{4}$

Apesar de raras, podem ter implicações graves uma vez que se estabelecem em pacientes idosos (mais frequentes no género feminino), com múltiplos fatores de risco e comorbilidades. Podem manifestar-se sob a forma de: oclusão intestinal baixa (cálculo impactado no cólon sigmoide), hemorragia digestiva baixa (erosão de vasos na parede do cólon), inflamação localizada/sépsis. ${ }^{5}$ Quando sintomáticas, a taxa de mortalidade é elevada (35\% - 40\%) e o cirurgião, pode ser chamado a intervir, num paciente de difícil abordagem clínico-cirúrgica, exigindo-se sensatez e alguma prudência. ${ }^{6-8}$

A inespecificidade clínica, a limitação dos meios auxiliares de diagnóstico, a polémica suscitada pelo tipo de tratamento mais adequado e a raridade desta entidade, motivaram a apresentação de um caso clínico elucidativo deste tipo de fístula biliodigestiva, expondo a sua conduta diagnóstica e terapêutica.

\section{CASO CLÍNICO}

Doente com 81 anos, do género feminino, diabética insulinotratada, hipertensa, com insuficiência cardíaca direita, hipertensão pulmonar grave e história de vários internamentos no Serviço de Medicina Interna por diabetes descompensada, enterites com diarreia que não cediam a medidas terapêuticas habituais, insuficiência cardíaca descompensada e insuficiência renal crónica.

Referia dor abdominal tipo cólica associada à paragem de emissão de gases e fezes.

Ao exame físico, a doente encontrava-se consciente, mas pouco colaborante, ligeiramente descorada e desidratada, encontrava-se hipotensa (100/66 mmHg), com ritmo cardíaco irregular, mas estável do ponto de vista hemodinâmico. Abdómen - ruídos hidro-aéreos aumentados em frequência e com timbre metálico, distendido, timpanizado, com dor difusa à palpação, mas sem sinais de irritação peritoneal.

A colocação de sonda nasogástrica, drenou de imediato, cerca de 21 de conteúdo fecaloide.

As determinações analíticas (hemograma e bioquímica evidenciaram hemoglobina 10,8 g/dL, leucócitos 10400 $\mathrm{U} / \mathrm{dL}$ (com neutrofilia), creatinina 2,87 mg/dL, ureia 32, K 3,2, Na 139, TGO 16 U, TGP 26 U, fosfatase alcalina, 288, INR 1,2.

Os exames de imagem (radiografias do tórax e abdómen, ecografia abdominal) demonstraram: alargamento do mediastino à custa da sombra cardíaca, níveis hidro-aéreos na topografia do intestino delgado e cólon, "distensão hídrica de muitas ansas do intestino delgado, com peristaltismo reduzido, com derrame peritoneal moderado, não se identificando a causa da obstrução". Para melhor esclarecimento do quadro clínico foi solicitada tomografia abdominal computorizada (sem contraste endovenoso): "é possível perceber a existência de uma imagem sugestiva de fístula colecistocólica (envolvendo o fundo da vesícula biliar e a metade direita do cólon transverso); identifica-se uma imagem sugestiva de cálculo biliar com $3,7 \mathrm{~cm}$ na transição do cólon descendente para a ansa sigmoide, onde neste segmento surgem múltiplos divertículos, com alterações que sugerem a 

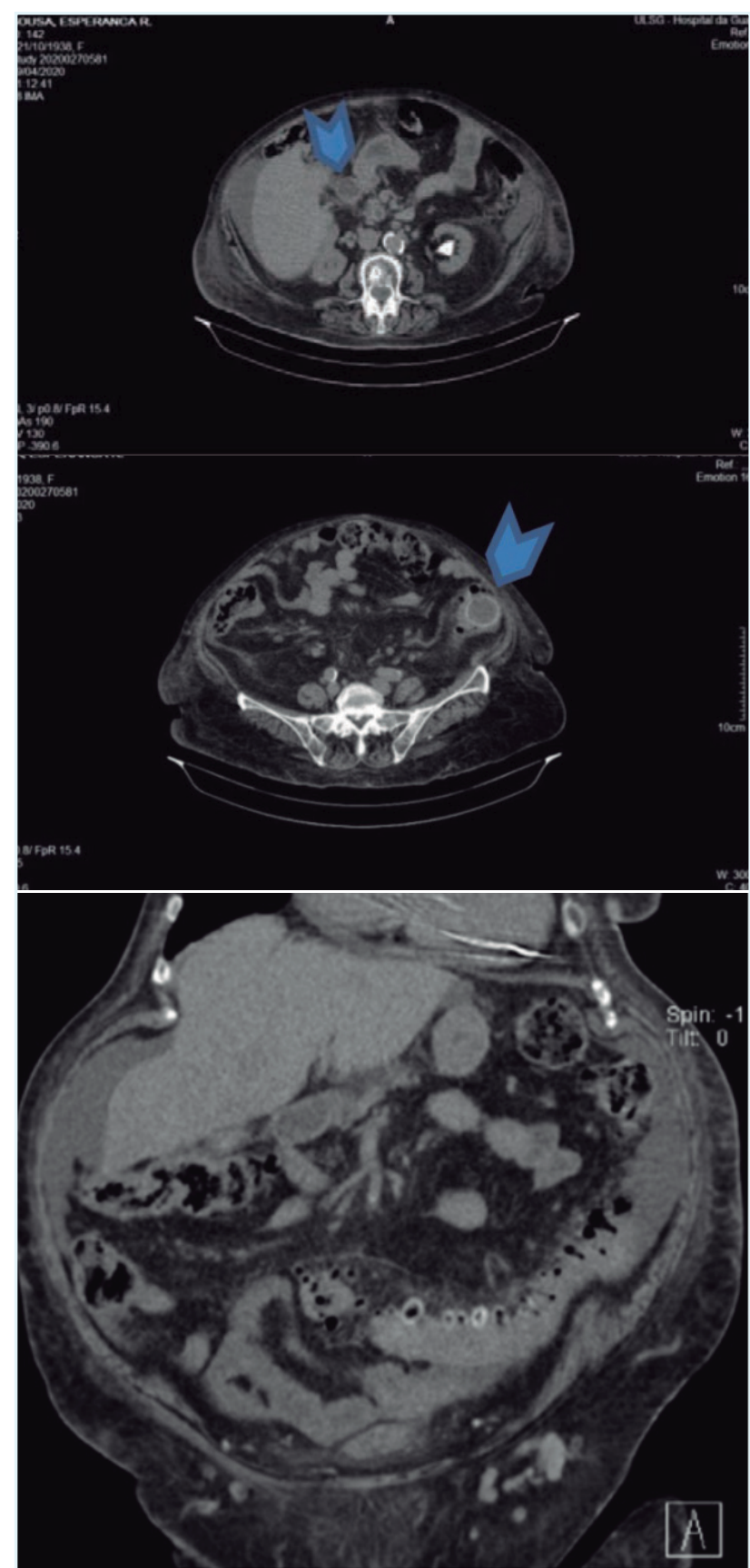

FIGURA 1. Tomografia computorizada (TC) abdominal com imagem sugestiva de fístula colecistocólica (setas).

existência prévia de processos inflamatórios locais (diverticulites), o que nos faz duvidar da possibilidade da passagem espontânea do cálculo através deste segmento"- (Fig. 1).

Perante os achados da tomografia, solicitou-se uma colonoscopia: "progressão até aos $35 \mathrm{~cm}$ e observado cálculo biliar a este nível, que ocupa praticamente todo o lúmen, embora se mobilize com as manobras de insuflação e aspiração"; área de mucosa com erosão a 30 cm; presença de numerosos divertículos a jusante do cálculo condicionando estreitamento do lúmen do cólon sigmoide e angulações acentuadas do mesmo, impossibilitando a remoção endoscópica do cálculo" - (Fig. 2).

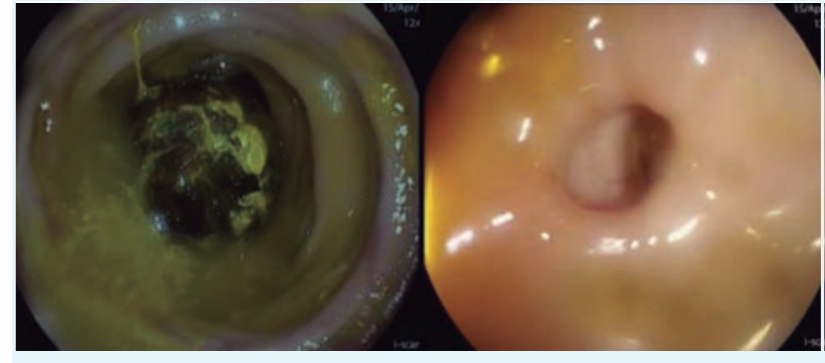

FIGURA 2. Colonoscopia com observação de cálculo biliar.

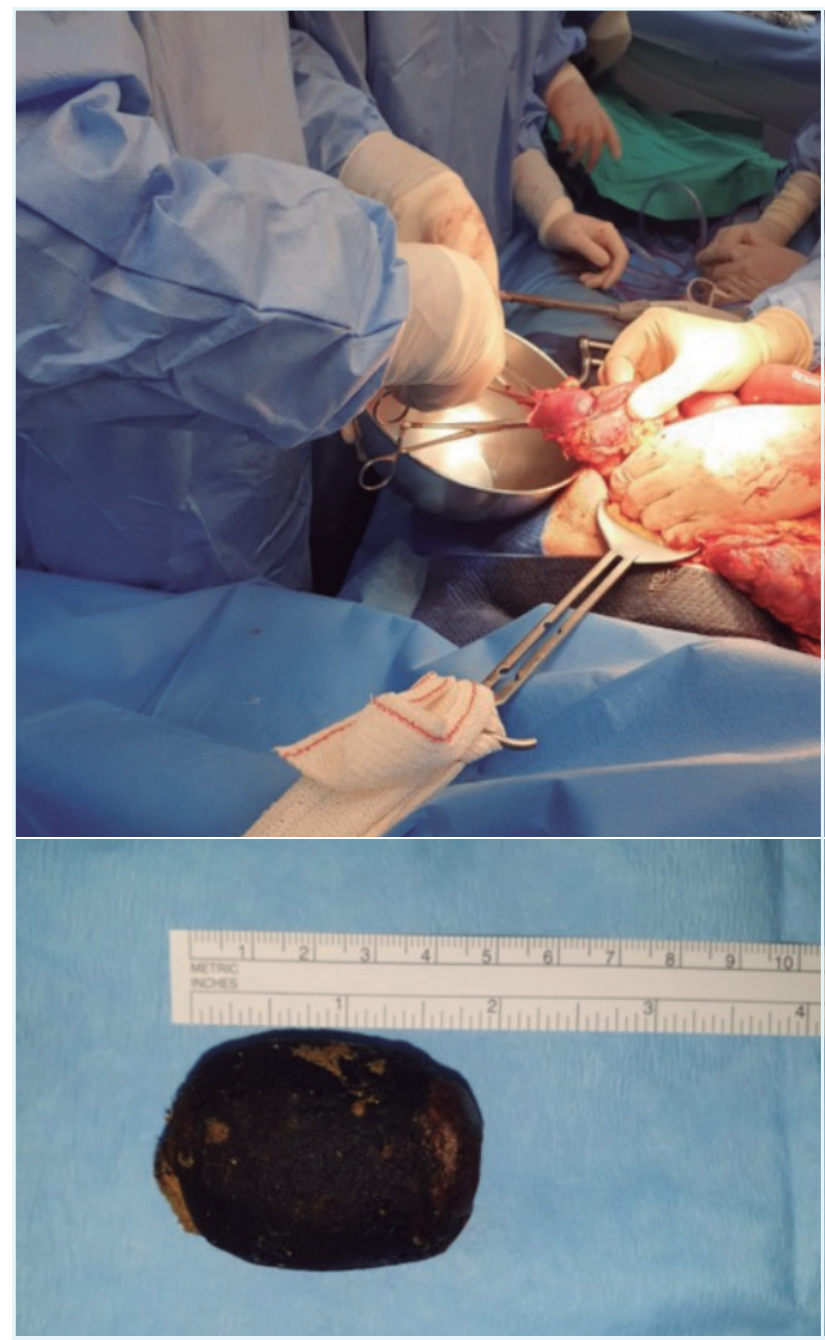

FIGURA 3. Procedimento de "milking" e medição do cálculo biliar.

Perante a tentativa infrutífera de remoção do cálculo por via endoscópica, submeteu-se a doente a uma celiotomia exploradora que evidenciou: oclusão intestinal baixa, condicionada por um cálculo impactado no cólon sigmoide deformado pela presença de divertículos e fibrose resultante de fenómenos de diverticulite prévios; fístula colecistocólica entre o ângulo hepático do cólon e a vesícula biliar.

Procedeu-se, à mobilização proximal do cálculo ("millking"), remoção através de colotomia e confeção de colostomia lateral sem intercorrências intraoperatórias (Fig. 3). 
No pós-operatório, verificou-se uma agudização da lesão renal crónica com acidémia metabólica, e necessidade de transferência para uma Unidade de Cuidados Intensivos deste Hospital e, posteriormente, para o Serviço de Nefrologia no Centro Hospitalar e Universitário de Coimbra.

\section{DISCUSSÃO}

O diagnóstico desta entidade exige um elevado índice de suspeição clínica em articulação com avaliação imagiológica e endoscópica complementares.

As atitudes terapêuticas, atualmente, aceites incluem:

- Remoção endoscópica do(s) cálculo(s) após fragmentação;

- Intervenção cirúrgica, por laparotomia ou laparoscopia, em um ou dois tempos para remoção do(s) cálculo(s) associada (um tempo) ou seguida (dois tempos) de colecistectomia e reparação da fístula.

A terapêutica cirúrgica num só tempo, permite o tratamento definitivo, eliminando teoricamente o risco de "íleus biliar" recorrente, a colangite, o carcinoma da vesícula biliar e a necessidade de outro procedimento cirúrgico. Não obstante, está associado a uma taxa de morbimortalidade mais elevada, pelo que deve ser reservado a pacientes com bom estado geral.

A escolha entre tratamento endoscópico e cirúrgico é condicionada fundamentalmente pelo material disponível, tamanho do(s) cálculo(s), estabilidade hemodinâmica e/ou opção do paciente. Nos doentes com risco cirúrgico elevado, o tratamento endoscópico surge como uma alternativa adequada pela menor taxa de complicações. Contudo, perante o seu fracasso é obrigatório o tratamento cirúrgico.

No caso clínico exposto o tratamento cirúrgico impôs-se perante o fracasso da terapêutica endoscópica. Uma vez realizada laparotomia verificou-se que as condições locais e o estado de equilíbrio hemodinâmico do doente não permitiam o tratamento definitivo em um só tempo: colecistectomia, reparação da fístula e remoção do cálculo. Optámos por manter a fístula "in situ" e proceder ao manuseamento do cálculo até ao cólon descendente através do processo de "ordenha" - "milking" e confecionar uma colostomia lateral atendendo à deformidade do cólon sigmoide condicionada pelos episódios repetidos de diverticulite prévios.

\section{CONCLUSÃO}

Apesar de raro, a possibilidade do "cólon biliar" existe, e desta vez sem solução endoscópica, numa doente com várias comorbilidades e atraso no diagnóstico (a doente estava internada há 5 dias no Serviço de Medicina Interna).

A conduta e terapêutica corretas são fundamentais para evitar a morbimortalidade elevada inerente. A eleição do tratamento adequado para cada caso clínico, endoscopia e/ou cirurgia, é função de fatores clínicos e técnicos.

Em doentes de alto risco, somos da opinião que o tratamento de eleição é a abordagem em dois tempos, isto é, resolução da oclusão intestinal em primeiro tempo, deixando a colecistectomia e tratamento da fístula para o segundo tempo, se a condição clínica da doente permitir.?

\section{RESPONSABILIDADESÉTICAS}

CONFLITOS DE INTERESSE: Os autores declaram a inexistência de conflitos de interesse na realização do presente trabalho.

FONTES DE FINANCIAMENTO: Não existiram fontes externas de financiamento para a realização deste artigo.

CONFIDENCIALIDADE DOS DADOS: Os autores declaram ter seguido os protocolos da sua instituição acerca da publicação dos dados de doentes.

CONSENTIMENTO: Consentimento do doente para publicação obtido.

PROVENIÊNCIA E REVISÃO POR PARES: Não comissionado; revisão externa por pares.

\section{ETHICAL DISCLOSURES}

CONFLICTS OF INTEREST: The authors have no conflicts of interest to declare.

FINANCING SUPPORT: This work has not received any contribution, grant or scholarship.

CONFIDENTIALITY OF DATA: The authors declare that they have followed the protocols of their work center on the publication of data from patients.

PATIENT CONSENT: Consent for publication was obtained.

PROVENANCE AND PEER REVIEW: Not commissioned; externally peer reviewed. 


\section{REFERÊNCIAS}

1. Jorge XJ, Sousa AL, Panão AE. Fístulas biliodigestivas litiásicas (a propósito de 3 casos clínicos). GE J Port Gastrenterol. 2013;20:119-22. doi: 10.1016/j.jpg.2012.07.002.

2. Tahir OM, Fedele CR, Sneider MB, Collins DS, Maloney DB. Cholecystocolonic fistula. Del Med J. 2014;86:373-5.

3. Conde LM, Tavares PM, Quintes JL, Chermont RQ, Perez MC. Laparoscopic management of cholecystocolic fistula. Arq Bras Cir Dig. 2014;27:285-7. doi: 10.1590/S010267202014000400013.

4. Inukai K. Gallstone ileus: a review. BMJ Open Gastroenterol. 2019:6:e000344. doi: 10.1136/bmigast-2019-000344.

5. Costi R, Randone B, Violi V, Scatton O, Sarli L, Soubrane O, et al. Cholecystocolonic fistula: facts and myths. A review of the 231 published cases. J Hepatobiliary Pancreat Surg. 2009;16:818. doi: 10.1007/s00534-008-0014-1.

6. Smyth J, Dasari BV, Hannon R. Biliary-colonic fistula. Clin Gastroenterol Hepatol. 2011;9:A26. doi: 10.1016/j. cgh.2011.04.015

7. Melo EL, Paula FT, Siqueira RA, Ribeiro SC. Cólon Biliar: "um caso incomum de oclusão intestinal. Radiol Brasil. 2015;48:127-8. doi: 10.1590/0100-3984.2014.0073.

8. Del Gaizo A, Bharet R. Cholecystocolonic fistula. Appl Radiol. 2006;35:21-2.

9. Rodríguez-Sanjuán JC, Casado F, Fernández MJ, Morales DJ, Naranjo A. Cholecystectomy and fistula closure versus enterolithotomy alone in gallstone ileus. Br J Surg. 1997;84:634-7. 